

\title{
Dinâmica espaço-temporal: condições atmosféricas versus manifestações de doenças respiratórias no Baixo Amazonas e Sudoeste do Pará
}

\author{
Maria Roseane Pereira dos Santos ${ }^{1}$, Welligton Conceição Da Silva², Éder Bruno Rebelo Da Silva ${ }^{3}$ \& \\ Ana Carla Dos Santos Gomes ${ }^{4}$ \\ ${ }^{1}$ Graduanda em Bacharelado Interdisciplinar em Ciências da Terra, Universidade Federal do Oeste do Pará, Rua Vera Paz, Bairro Salé, S/N, \\ CEP 68040-470, Santarém (PA), Brasil, Tel.: +55 (015 93) 991245612, E-mail: roseanemaria022@ gmail.com (Autor \\ correspondente). ${ }^{2}$ Programa de Pós-graduação em Saúde e Produção Animal da Amazônia, Universidade Federal Rural da Amazônia, Estrada \\ principal da UFRA, Bairro Curió Utinga, 2150, CEP 66077-830, Belém (PA), Brasil, Tel.; +55 (015 93) 991408094, E-mail: \\ welligton.medvet@gmail.com. ${ }^{3}$ Programa de Pós-graduação em Agronomia, Universidade Federal Rural da Amazônia, Av. Barão de \\ Capanema, Bairro Caixa d’água, S/N, CEP 68700-665, Capanema (PA), Brasil, Tel.: +55 (015 93) 991105326, E-mail: \\ eder.b.rebelo@gmail.com. ${ }^{4}$ Instituto de Engenharia e Geociências, Universidade Federal do Oeste do Pará, Rua Vera Paz, Bairro Salé, S/N, \\ CEP 68040-470, Santarém (PA), Brasil, Tel.: +55 (015 93) 992203437, E-mail: anacarlasg02@ gmail.com.
}

Artigo recebido em 28/06/2021 e aceito em 13/09/2021

\begin{abstract}
R E S U M O
O clima pode influenciar no aparecimento de ambientes propícios a proliferação de vetores de doenças respiratórias, além de influenciar enfermidades e agravos relacionados pelos fenômenos climáticos. Assim, o objetivo neste estudo foi verificar a dinâmica espaço-temporal entre a ocorrência de condições atmosféricas e as manifestações de doenças respiratórias no baixo amazonas e sudoeste do Pará. Adotou-se o período de 2008 a 2020 para os municípios de Belterra e Monte Alegre (Baixo Amazonas) e Itaituba (Sudoeste). Utilizou-se dados meteorológicos, disponibilizados pela plataforma digital do Instituto Nacional de Meteorologia (INMET) e os dados de doenças respiratórias por meio do Departamento de Informática do Sistema Único de Saúde (DATASUS). Coletou-se dados de paciente adultos, jovens e crianças (única amostra). Empregou-se o modelo de Regressão Linear Múltipla via análise de componente principal (ACP), objetivando avaliar a possível associação entre as manifestações de doenças respiratórias e as variáveis meteorológicas, sendo as análises realizadas no software estatístico livre R. Observou-se que houve correlação linear significativa $(\mathrm{p}<0,05)$ entre o período menos chuvoso e os casos de doenças respiratórias em Belterra e Monte Alegre. Em Monte Alegre foi possível evidenciar correlação significativa do chuvoso para o menos chuvoso. Já em Itaituba não se observou correlação entre as estações e doenças respiratórias. Por fim, em Belterra, Itaituba e Monte alegre o número de internações por doenças respiratórias apresentou aumento no período de transição, especificamente no período de junho a agosto.
\end{abstract}

Palavras-chave: clima, saúde, precipitação pluviométrica

\section{Spatio-temporal dynamics: atmospheric conditions versus manifestations of respiratory diseases in the Lower Amazon and the Southwest of Pará}

\begin{abstract}
A B S T R A C T
The climate can influence the non-appearance of environments conducive to the proliferation of vectors of respiratory diseases, in addition to influencing diseases and conditions related to climatic phenomena. In addition, the objective of this study was to verify the spatio-temporal dynamics between the occurrence of atmospheric conditions and the manifestations of respiratory conditions outside the Amazon and southwestern Pará. The period from 2008 to 2020 was adopted for the municipalities of Belterra and Monte Alegre (Baixo Amazonas) and Itaituba (Southwest). Use of meteorological data, made available on the digital platform of the National Institute of Meteorology (INMET) and respiratory education data through the Department of Informatics of the Unified Health System (DATASUS). Data were collected from adult, young and infant patients (sample only). Model of Multiple Linear Regression or Regression using principal component analysis (PCA), aiming to validate a possible association between the manifestations of respiratory conditions and different meteorological conditions, with the analyzes being carried out without free statistical software R. Note that there is a linearly significant correlation (p $<0.05)$ between the least humid period in cases of respiratory diseases in Belterra and Monte Alegre. In Monte
\end{abstract}

Santos., M., R., P., Silva., W., C., Silva., É., B., R., Gomes., A., C., S. 
Alegre it was possible to show a significant correlation between cool and less cool. In Itaituba, no correlation was observed between seasons and respiratory conditions. Finally, in Belterra, Itaituba and Monte Alegre, the number of hospitalizations for respiratory doses seems to increase, not the transition period, specifically the period from June to August.

Keywords: climate, health, rainfall

\section{Introdução}

As condições meteorológicas exercem total influência no desempenho das atividades realizadas no cotidiano da humanidade. Entretanto, a dinâmica climática, ambiental e condições socioeconômicas, passaram a se modificar rapidamente, devido ao processo de globalização, urbanização e crescimento das cidades (Gonçalves e Coelho, 2010; Dapper et al., 2016; Moraes et al., 2019). Dessa forma, esses efeitos vieram acompanhados das desigualdades sociais e ambientais nos centros urbanos, que influenciaram na saúde e qualidade de vida de seus habitantes. Isso se deve ao favorecimento do surgimento e agravamento de diversas doenças (Nogueira et al., 2011; Silva, et al., 2014; Ribeiro e Vargas, 2015; Sales et al., 2018; Martins et al., 2019; Linares et al., 2020).

As variações de temperatura do ar e de outras variáveis meteorológicas, tais como umidade relativa do ar e precipitação, são grandes responsáveis ambientais para $\mathrm{o}$ aumento do risco de internações e de mortes resultantes de doenças respiratórias no mundo (Dadbakhsh et al., 2017; Moraes et al., 2019; Lima et al., 2021). Dessa forma, a funcionalidade dos pulmões pode ser afetada pela umidade relativa dor ar e precipitação, assim como a temperatura, facilitando $o$ aparecimento de casos de gripes, rinites, bronquites e a piora dos casos crônicos de asma, pois ajudam no surgimento e crescimento de vírus, bactérias, mofos, fungos e alérgenos (Royé et al., 2015; Lam et al., 2016; Alves e Freitas, 2021).

As doenças respiratórias são infecções que ocasionam obstrução na passagem de ar a nível do trato respiratório superior e inferior, fazendo com que grande parte das infecções apresente elevada taxa de óbito no mundo (Thomazelli, 2007; Santana et al., 2020; Yang et al., 2020). Nesse contexto, existem estudos que evidenciam a relação direta entre as variações meteorológicas e a saúde da população, principalmente no comprometimento das funções pulmonar e no aparecimento de doenças no trato respiratório (Carvalho, 2016; Liang et al., 2016; Yi et al., 2019).

Infecções respiratórias possuem alta taxa de contágio, devido seu grande potencial de propagação que ocorre pelas gotículas contaminadas, podendo ser liberadas pelo bocejo, espirro, através da fala e pela transmissão do contato da mão com superfícies infectadas (Setti et al., 2020). Para Silva et al. (2017) as infecções respiratórias são um grande desafio para os serviços de saúde, visto que a doença é uma das principais causas de morte por incapacidade no Brasil.

Variações de umidade e temperatura são consideradas variáveis relacionadas com as alterações nas concentrações de poluentes, principalmente em áreas urbanas onde a dispersão dessas partículas é favorecida pela presença de ventos e precipitações, que prolonga $\mathrm{o}$ período desses poluentes na atmosfera, resultando em internações por doenças respiratórias (Andreão et al., 2019). Dessa forma, a elevação da temperatura associa-se a grande elevação da quantidade de partículas inaláveis (César, 2016; Manisalidis et al., 2020). Assim, tanto a alta na temperatura quanto a diminuição brusca são relacionadas aos casos de doenças respiratórias (Briggs, 2003; Souza et al., 2012).

O clima pode influenciar no aparecimento de ambientes propícios a proliferação de vetores de doenças respiratórias, além de influenciar enfermidades e agravos relacionados pelos fenômenos climáticos (Barcellos e Hacon, 2016; Galvão e Leite, 2017; Fernandes et al., 2020). Dessa forma, faz-se necessário relacionar os mecanismos de exposição, ou seja, as variáveis climáticas ao surgimento de doenças e como esse processo ocorre. Assim, a hipótese neste estudo é que as variáveis meteorológicas influenciam no índice de doenças respiratórias.

Com base nessas informações, o objetivo neste estudo foi verificar a dinâmica espaço-temporal entre as condições atmosféricas e manifestações de doenças

Santos., M., R., P., Silva., W., C., Silva., É., B., R., Gomes., A., C., S. 
respiratórias em municípios do Baixo Amazonas e Sudoeste do Pará.

\section{Área, material e métodos}

Área de estudo

O estudo foi realizado nos municípios de Belterra e Monte Alegre pertencentes a mesorregião do Baixo Amazonas e no município de Itaituba que se localiza no Sudoeste paraense (Fig. 1), no qual foram coletados dados mensais de janeiro a dezembro no período de 2008 a 2020 nos municípios de Belterra, Itaituba e Monte Alegre.

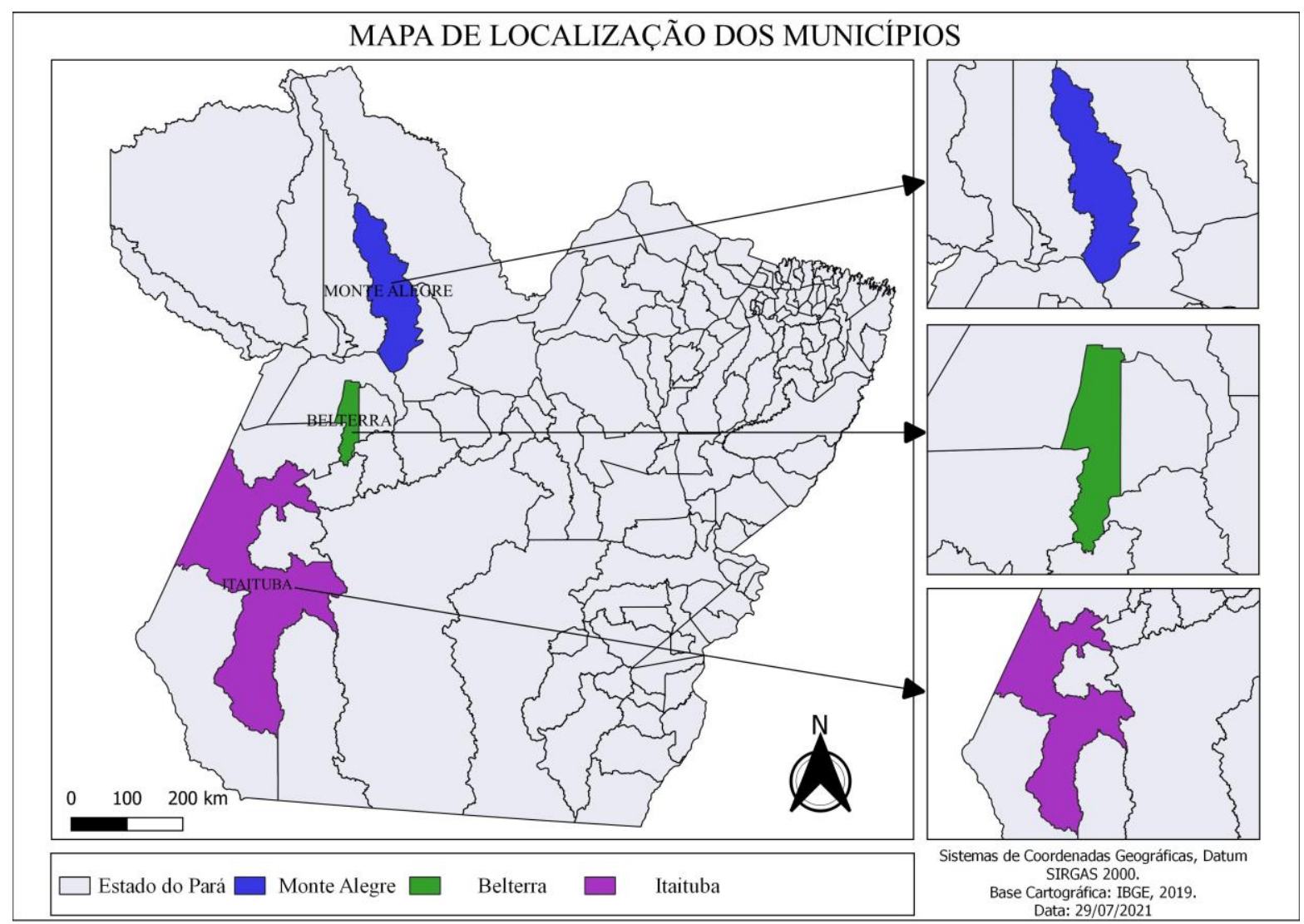

Figura 1. Mapa de localização geográfica dos municípios estudados.

Os três municípios possuem clima predominante tropical quente e úmido, do tipo Am conforme a classificação climática instituída por Koppen e Geiger (1928). Na
Tabela 1 são apresentadas as informações referentes às coordenadas geográficas e condições climáticas dos três diferentes parâmetros selecionados.

Tabela 1. Dados referentes às coordenadas geográficas e informações meteorológicas dos municípios estudados.

\begin{tabular}{cccccc}
\hline Município & Latitude (S) & Longitude $(\mathrm{W})$ & Altitude $(\mathrm{m})$ & $\mathrm{UR}(\%)$ & $\mathrm{T}_{\mathrm{m}}\left({ }^{\circ} \mathrm{C}\right)$ \\
\hline Belterra & $02^{\circ} 38^{\prime} 11^{\prime}$, & $54^{\circ} 56^{\prime} 18^{\prime}$ & 152 & 86 & 28 \\
\hline Itaituba & $04^{\circ} 16^{\prime} 34^{\prime}$, & $55^{\circ} 59^{\prime} 01^{\prime}$ & 18 & 87 & 26,6 \\
\hline $\begin{array}{c}\text { Monte } \\
\text { Alegre }\end{array}$ & $01^{\circ} 59^{\prime} 56^{\prime}$, & $54^{\circ} 4^{\prime} 58^{\prime \prime}$ & 59 & 80 & 27,4 \\
\hline
\end{tabular}

Nota: $\mathrm{UR}=$ umidade relativa; $\mathrm{Tm}=$ Temperatura média do ar.

Dados meteorológicos

Foram coletados dados de temperatura máxima, mínima e média do ar, umidade relativa do ar e precipitação pluviométrica para o período correspondente de 2008 a 2020, por meio da plataforma digital do Instituto Nacional de Meteorologia (INMET), proveniente de estações convencionais, registrados na

Santos., M., R., P., Silva., W., C., Silva., É., B., R., Gomes., A., C., S. 
Organização Meteorológica Mundial (OMM) com número de registro 82246 (Belterra), 82445 (Itaituba) e 82181(Monte Alegre).

Em relação à sazonalidade, constata-se que a estação menos chuvosa ocorre nos meses de junho a novembro, e a chuvosa ocorre entre os meses de dezembro a maio (Correa et al., 2016). Entre essas estações evidenciou-se o período de transição, que diz respeito ao momento em que ocorre a troca do período chuvoso para o menos chuvoso, e, do período menos chuvoso para o chuvoso, ambas acontecem na região entre os meses de maio e junho, e, novembro e dezembro, respectivamente.

\section{Dados de Saúde}

Coletou-se dados em relação ao número de atendimentos (ano/mês) de pessoas internadas por doenças respiratórias (IDR) listadas no Capítulo X (CID-10), sob código J00-J99, através da busca na plataforma informações e saúde (TABNET) fornecidos pelo Departamento de Informática do Sistema Único de Saúde (DATASUS), por meio da plataforma digital do Ministério da Saúde. Estes dados eram provenientes de paciente adultos, jovens e crianças, considerando-os como uma única amostra.

\section{Análise estatística}

A etapa inicial ocorreu por meio da estatística descritiva que nos permite organizar, resumir e descrever o conjunto de dados. Com o propósito de avaliar a possível associação entre as internações por doenças respiratórias (IDR) e as variáveis meteorológicas utilizou-se o modelo de Regressão Linear Múltipla via análise de componente principal (ACP) para evitar problema de multicolinearidade.

$\mathrm{Na} \mathrm{ACP}$, combinações lineares das variáveis independentes são formadas. A primeira componente principal é a combinação que explica a maior contribuição para a variância na amostra. A segunda componente principal explica a segunda maior contribuição para a variância, sem estar correlacionada com a primeira. Sucessivas componentes explicam porções progressivamente menores da variância da amostra total, sem apresentar correlação com as componentes anteriores (Hair et al., 2005). Segundo Dantas et al. (2016) a análise de regressão é uma importante técnica estatística, que tem sido utilizada em aplicações de diversas áreas e é adequada quando se deseja estudar o comportamento de uma variável resposta em relação a outras (explicativas) que contribuem para a sua variabilidade. Sua base estatística vem da regressão linear simples, que se restringe a duas variáveis e a apenas uma equação funcional do primeiro grau $(\mathrm{Y}=\mathrm{a}+$ $b X)$.

Segundo Wilks (2006) a definição matemática do modelo de regressão múltipla incide de uma função linear (equação 1):

$$
\begin{array}{rr}
Y=\beta 0+\beta 1 X 1 \\
+\beta 2 X 2+\ldots+\beta b k X k+\varepsilon & \text { (eq.1) }
\end{array}
$$

onde Y é a variável dependente; $ß 0$ corresponde a um coeficiente técnico fixo, a um valor de base a partir do qual começa Y; ßk corresponde aos coeficientes técnicos atrelados às Variáveis Independentes; Xk as Variáveis Independentes e $\varepsilon$ é o erro aleatório.

Neste estudo foi considerado a variáveis de resposta as IDR e as variáveis meteorológicas o conjunto de variáveis explicativas. Todas as técnicas mencionadas foram realizadas por meio do software estatístico livre R, versão 4.0.0.

\section{Resultados e discussão}

Na tabela 2 observa-se os dados da estatística descritiva relacionadas as variáveis meteorológicas e número de internações por doenças respiratórias por município. O maior índice de precipitação pluviométrica foi registrado em Belterra $(737.10 \mathrm{~mm})$, bem como a temperatura máxima $\left(35.35^{\circ} \mathrm{C}\right)$. Já temperatura mínima teve o menor registro em Monte Alegre, com $21.00^{\circ} \mathrm{C}$. Já a Umidade relativa apresentou máxima de $92.79 \%$ em Belterra e a mínima em Itaituba $\left(64.00^{\circ} \mathrm{C}\right)$. Por fim, o maior número de internações foi registrado em Itaituba com 239.000 casos. Assim, vale considerar também que este foi o município que apresentou maior amplitude térmica com $13.98^{\circ} \mathrm{C}$, seguido de Belterra com $13.68^{\circ} \mathrm{C}$ e Monte Alegre com $13.41^{\circ} \mathrm{C}$. Já em relação aos dados pluviométricos, o município de Belterra apresentou maior variabilidade de precipitação ao comparar com os demais municípios estudados. 
Tabela 2. Estatísticas Descritivas das variáveis meteorológicas e as doenças respiratórias nos municípios de Belterra, Monte Alegre e Itaituba, no estado do Pará, no período de 2008 a 2020.

\begin{tabular}{|c|c|c|c|c|}
\hline \multicolumn{4}{|c|}{ Belterra } & \\
\hline $\begin{array}{l}\text { PRECIPITAÇÃO } \\
(\mathrm{mm})\end{array}$ & $\operatorname{TMAX}\left({ }^{\circ} \mathrm{C}\right)$ & TMIN $\left({ }^{\circ} \mathrm{C}\right)$ & UR $(\%)$ & $\begin{array}{l}\mathrm{DR}\left(\mathrm{N}^{\circ} \mathrm{de}\right. \\
\text { Internações })\end{array}$ \\
\hline Min.: 0.00 & Min.: 29.23 & Min.: 21.67 & Min.: 78.08 & Min.: 0.000 \\
\hline Median: 129.15 & Median: 31.25 & Median: 22.26 & $\begin{array}{l}\text { Median: } \\
88.68\end{array}$ & Median: 6.000 \\
\hline Mean: 159.91 & Mean: 31.56 & Mean: 22.34 & Mean: 87.66 & Mean: 7.551 \\
\hline Max.: 737.10 & Max.: 35.35 & Max.: 23.41 & Max.: 92.79 & Max.: 40.000 \\
\hline \multicolumn{5}{|c|}{ Monte Alegre } \\
\hline Min.: 0.0 & Min.: 29.19 & Min.: 21.00 & Min. :65.00 & Min.: 5.00 \\
\hline Median: 116.0 & Median: 31.18 & Median: 23.36 & $\begin{array}{c}\text { Median } \\
: 78.00\end{array}$ & Median: 27.50 \\
\hline Med.: 152.9 & Med.: 31.56 & Med: 23.26 & Med :76.94 & Med: 30.97 \\
\hline Max.: 673.6 & Max.: 34.41 & Max.: 24.95 & Max. :86.00 & Max.: 100.00 \\
\hline \multicolumn{5}{|c|}{ Itaituba } \\
\hline Min.: 4.5 & Min.: 30.59 & Min.: 23.09 & Min.: 64.00 & Min.: 3.00 \\
\hline Median: 163.7 & Median: 33.19 & Median: 24.38 & $\begin{array}{l}\text { Median: } \\
83.00\end{array}$ & Median: 110.00 \\
\hline Med.: 187.2 & Med.: 33.46 & Med.: 24.41 & Med: 81.20 & Med.: 98.51 \\
\hline Max.: 494.7 & Max.: 37.07 & Max.: 26.02 & Max.: 89.00 & Max.: 239.00 \\
\hline
\end{tabular}

Na Figura 2A é possível identificar que a temperatura máxima do ar foi superior a $35^{\circ} \mathrm{C}$ nos meses de outubro e novembro no município de Belterra. Em relação à temperatura mínima esta foi inferior a $25^{\circ} \mathrm{C}$, alcançado $23^{\circ} \mathrm{C}$ em setembro, outubro, novembro e dezembro (Figura 2B). Em relação aos dados pluviométricos, constatou-se que no período de janeiro a setembro, com exceção do mês de abril, houve maior índice de precipitação no município de Belterra, com máxima de $500 \mathrm{~mm}$ (Figura 2C). A umidade relativa do ar foi superior a $90 \%$ nos meses de janeiro, fevereiro, março, abril, maio, junho e dezembro (Figura 2D).

Em relação a temperatura máxima observou-se nos meses de janeiro, março, abril, setembro e dezembro valores acima da média em Belterra, sendo os valores de temperatura mínima acima da média, outliers, evidenciado nos meses de janeiro, fevereiro e agosto, nos meses de setembro e maio abaixo da média.

Em relação à precipitação no município de Belterra, notou-se que os meses de fevereiro, julho, agosto e setembro apresentaram valores acima da média, por outro lado o mês de outubro estava com valores acima e abaixo da média. Outliers também foram evidenciados nos valores de umidade relativa do município, constatando que o mês de outubro obteve valor acima da média, seguido pelos meses de janeiro e maio que apresentaram valores abaixo da média e, por fim, o mês de março que apresentou valores acima e abaixo da média.

No município de Monte Alegre a temperatura máxima apresentou valores discrepantes de média nos meses de janeiro, fevereiro, março e julho. Se tratando de temperatura mínima os meses de fevereiro, março, abril, maio e junho ficaram abaixo da média, ressaltando que nos meses de fevereiro e maio os valores foram acima e abaixo da média. A variável precipitação apresentou valores acima da média nos meses de abril, outubro e novembro e os valores de umidade relativa do ar mostram outliers nos meses de abril, agosto e setembro, com valores abaixo da média no mês de janeiro.

Santos., M., R., P., Silva., W., C., Silva., É., B., R., Gomes., A., C., S. 
Por fim, o município de Itaituba apresentou valores fora da média de temperatura máxima nos meses de janeiro, fevereiro, abril, maio, julho, agosto e dezembro, sendo os meses acima da média, junho e setembro. A temperatura mínima demonstrou

A

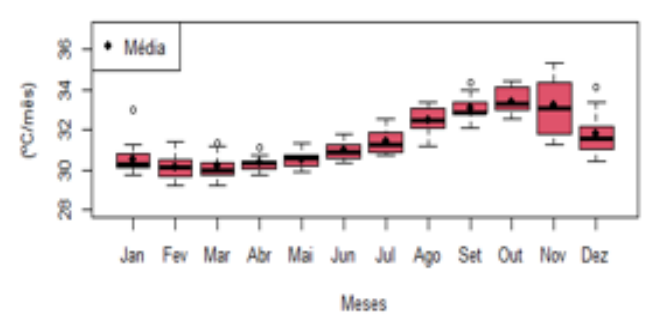

B

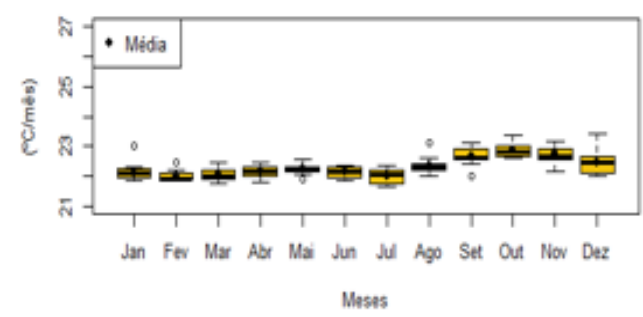

valores acima da média nos meses de junho e setembro e janeiro acima e abaixo da média. Em relação à umidade relativa do ar, os meses de fevereiro, julho, setembro e dezembro ficaram abaixo da média e o mês de outubro apresentou valores acima e abaixo da média.

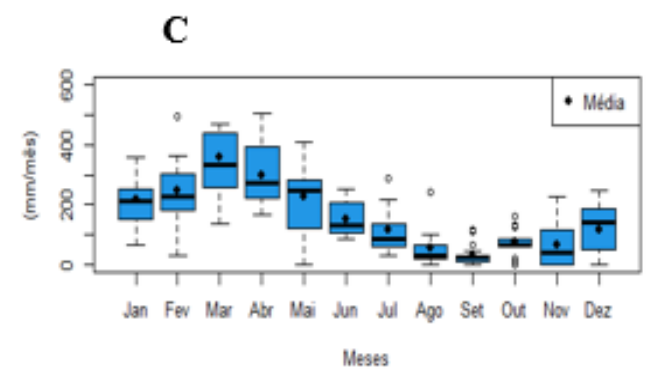

D

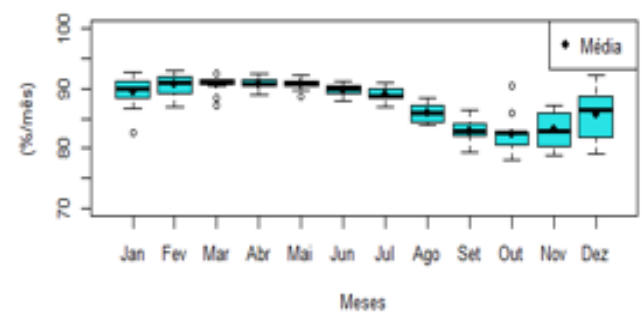

Figura 2. Boxplot mensal das variáveis meteorológicas de Belterra, no estado do Pará, no período de 2008 a 2020, onde: A) Temperatura máxima do ar, B) Temperatura mínima do ar, C) Precipitação e D) Umidade relativa do ar.

Em Monte alegre a temperatura máxima foi inferior a $35^{\circ} \mathrm{C}$ durante todos os meses do ano, atingindo no máximo $34^{\circ} \mathrm{C}$ (Figura 3A). Em relação a temperatura mínima esta foi inferior a $25^{\circ} \mathrm{C}$, alcançando $24^{\circ} \mathrm{C}$ em fevereiro (Figura 3B). A precipitação pluviométrica atingiu máxima superior $650 \mathrm{~mm}$ em maio (Fig. 3C). De acordo Nechet (1997), o aumento da temperatura tem relação considerável entre a temperatura mínima do ar e os casos de problemas respiratórios, sendo o período chuvoso intrinsicamente relacionado ao período dos altos índices de casos por infecções virais. A umidade relativa do ar apresentou valores inferiores a $90 \%$, em todos os meses do ano (Figura 3D).

Ao comparar o período chuvoso com o menos chuvoso, fica perceptível a exposição a alta umidade relativa do ar, associada ao maior tempo em que as pessoas habitualmente se mantêm em seus ambientes domiciliares fechados tendo menor arejamento do mesmo e com a baixa incidência de raios solares no ambiente interno, contribuindo desta forma para o alojamento de fungos e mofos, fatores estes que influenciam para o aumento de doenças respiratórias, principalmente as alérgicas (Mold, 2008).

Na pesquisa desenvolvida por Atkinson et al. (2006) e Dales et al, (2003) a proliferação de fungos no ambiente durante o período chuvoso também foi considerado um fator que pode predispor a ocorrência de doenças respiratórias, visto que estes observaram uma correlação entre os esporos de fungos e complicações por asma nos Estados Unidos. Já no Brasil, no Distrito Federal, Valença et al. (2006) identificaram a prevalência no número de atendimentos de emergência por asma em crianças em períodos de maior precipitação, provavelmente acometidos pela presença de microrganismos no ambiente.

Santos., M., R., P., Silva., W., C., Silva., É., B., R., Gomes., A., C., S. 
A

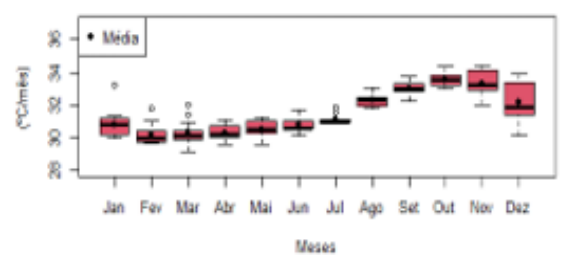

B

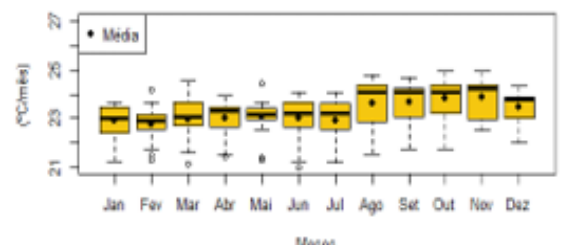

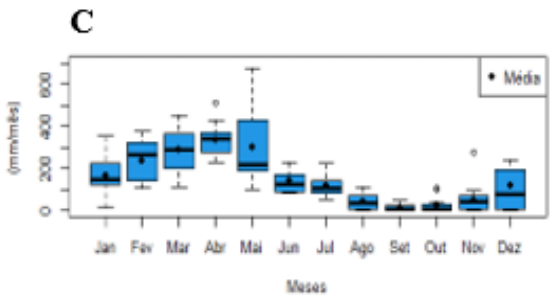

D

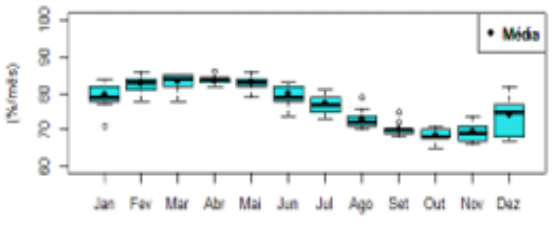

Woses

Figura 3. Boxplot mensal das variáveis meteorológicas de Monte Alegre, no estado do Pará, no período de 2008 a 2020, onde: A) Temperatura máxima do ar, B) Temperatura mínima do ar, C) Precipitação e D) Temperatura média.

Em Itaituba a temperatura máxima foi superior a $36^{\circ} \mathrm{C}$ em agosto (Figura $4 \mathrm{~A}$ ). E a temperatura mínima apresentou valores superiores a $25^{\circ} \mathrm{C}$, nos meses de janeiro, março, abril, maio, outubro, novembro e dezembro (Figura 4B). Em relação aos dados pluviométricos, em Itaituba, identificou-se que os valores de precipitação atingiram máxima inferior a $500 \mathrm{~mm}$ durante todos os meses do ano (Figura 4C). Com relação a umidade relativa do ar constatou-se que valores de média foi superior a $90 \%$ em Itaituba, especificamente, nos meses de janeiro, março, abril, maio, junho, julho, agosto e setembro (Figura 4D).

\section{A}

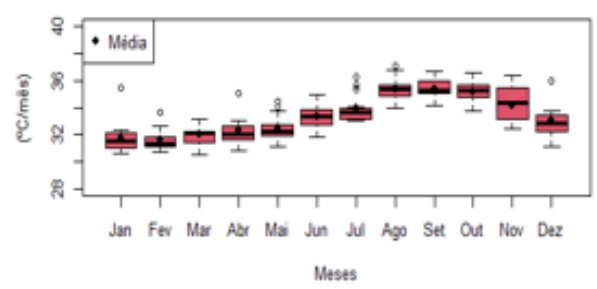

B

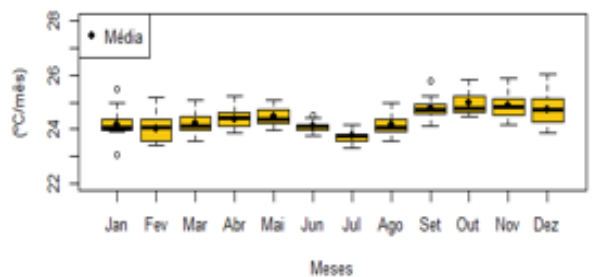

C

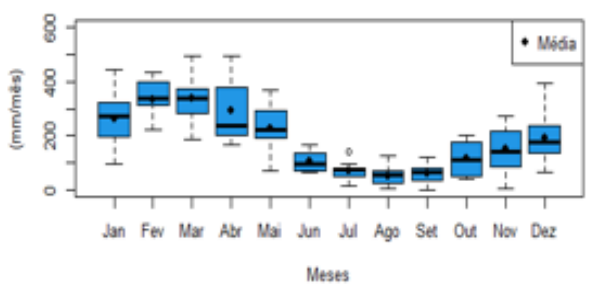

D

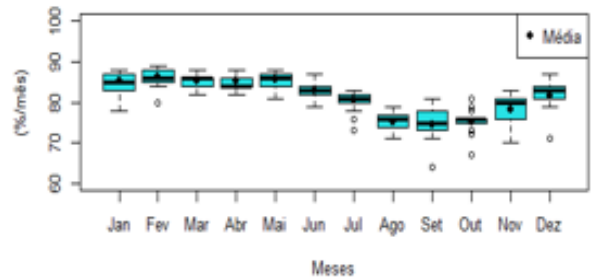

Figura 4. Boxplot mensal das variáveis meteorológicas em Itaituba, no estado do Pará, no período de 2008 a 2020, onde: A) Temperatura máxima do ar, B) Temperatura mínima do ar, C) Precipitação e D) Temperatura média.

Santos., M., R., P., Silva., W., C., Silva., É., B., R., Gomes., A., C., S. 
$\mathrm{Na}$ Figura 5, observa-se que em Belterra e Itaituba o maior número de internações por doenças respiratórias, foi registrado no período de transição (junho a agosto). Entretanto em Monte Alegre o maior índice se concentrou entre maio e junho. Porto e Latuf (2017), ressaltam que o período de transição afeta os valores de internações, quando há mudança no comportamento de precipitação, da estação chuvosa para a menos chuvosa, ocasionando um expressivo aumento, justificando que a população fica mais vulnerável às oscilações deste período.

Os resultados do presente estudo, corroboram com os achados descritos por Gomes et al. (2016) no município de SantarémPará no ano de 2010, que registraram o maior número de internações por doenças respiratórias no mês de junho (período de transição entre o mais chuvoso para o menos chuvoso) em crianças, o qual é caracterizado por apresentar maiores temperaturas, diminuição no regime de chuvas e da umidade relativa e aumento da precipitação atmosférica.

Os meses com maior número de casos de pessoas acometidas por doenças respiratórias no município de Belterra, Monte Alegre e Itaituba, foram em julho, maio, junho, respectivamente. Destaca-se que em Belterra a média de casos foi superior a 20, Monte alegre inferior a 110 casos e em Itaituba superior a 200 casos.

Avaliando o número de casos por doenças respiratórias em crianças no município de Campina grande e Monteiro, Azevedo et al. (2015) constaram que os meses de junho e julho foram os que apresentaram maior índice de infecções respiratórias agudas. Apresentando resultados diferentes do presente estudo, no qual se observou o maior número de casos em agosto. Isso pode explicado, devido, a diferença climatológica das regiões estudadas, onde em Campina grande e Monteiro o período de junho e julho é caracterizado com maior índice pluviométrico ao comparar com os municípios estudados.

Com relação aos índices de precipitação e os casos de doenças respiratórias, por exemplo, a pneumonia, diferentes estudos apontam uma associação entre ambas, sobretudo em ambientes tropicais, locais em que a sazonalidade das precipitações pode ser mais predominante que as oscilações térmicas (Chan et al., 2002; Moura et al., 2006; Paynter et al., 2010).
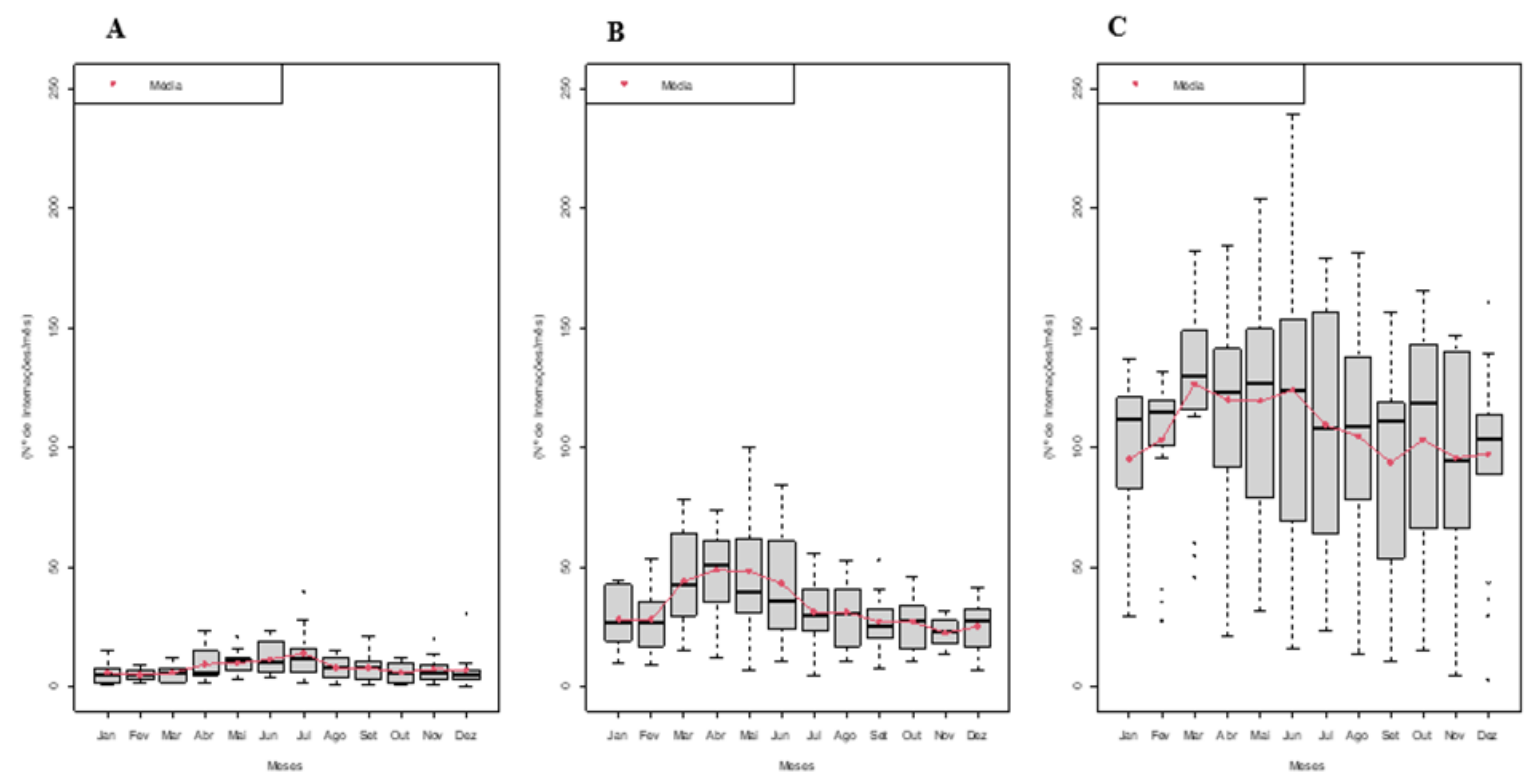

Figura 5. Boxplot mensal do número de internações por doenças respiratórias nos municípios de: A) Belterra, B) Monte Alegre e C) Itaituba, no estado do Pará, no período de 2008 a 2020.

Santos., M., R., P., Silva., W., C., Silva., É., B., R., Gomes., A., C., S. 
Avaliando os dados deste estudo, foi possível constatar a presença de Outliers em alguns dados deste estudo. Entretanto, ressaltase que neste estudo não se objetivou esclarecer as causas que ocasionaram a presença desses valores discrepantes. Isso pode ocorrer, possivelmente, devido às condições climáticas.

Observa-se que o número de internações por doenças respiratórias entre os meses de janeiro a maio nos três municípios estudados, são reduzidas se comparadas aos meses de junho a agosto. De acordo com Passos et al. (2014) isto pode ser explicado porque durante a estação chuvosa, as baixas temperaturas tendem a diminuir a funcionalidade do epitélio nasal por alteração celular e redução dos mecanismos de defesa mucociliar, em consequência disto o trato respiratório tanto superior quanto inferior ficam mais susceptíveis aos processos infecciosos, acometendo a população á adquirirem essas complicações durante este período. Apresentando maior pico de incidência no número de internações a partir do segundo semestre do ano pelo alto índice de pessoas infectadas durante o primeiro semestre.

Segundo Ignotti et al. (2010) o clima quente associado a altas temperaturas é um fator determinante para o surgimento de queimadas na Amazônia e do consequente aumento da poluição atmosférica, favorecendo a ocorrência de doenças respiratórias alérgicas. Corroborando com os dados obtidos no presente estudo, Fernando et al. (2018) descrevem que os municípios situados entre a mesorregião do sudeste e sudoeste expõe altos índices de focos de calor devido á incêndios associados a alteração do uso da terra.

Corroborando com Neto et al. (2003) que relatam o aumento de doenças virais em regiões que apresentam clima tropical, ainda segundo os autores a epidemiologia das viroses pode acontecer após mudanças nos padrões climáticos, como por exemplo o período mais chuvoso, para o menos chuvoso, ou seja, no período de transição, provocando a instalação de doenças respiratórias. Com resultados semelhantes ao observado neste estudo, Conceição et al. (2015) em Vitória da Conquista no estado da Bahia, também identificaram casos clínicos compatíveis com viroses em todos os meses do ano, evidenciando um maior índice de casos entre os meses de março e setembro, com o maior pico em agosto. Com resultados diferentes ao obtidos nesse estudo Picanço e Gomes (2019) em Belém, Pará, observaram que o maior índice de pacientes com síndromes respiratórias ocorreu no mês de setembro.

A correlação entre os períodos menos chuvosos apresentou correlação estatística $(p<0,05)$ com os casos de doenças respiratórias nos municípios de Belterra e Monte Alegre. Além disso, em Monte também se observou correlação no período de transição correspondente do chuvoso para menos chuvoso $(\mathrm{p}<0,05)$. Já em Itaituba não se observou correlação entre as estações e doenças respiratórias ( $\mathrm{p}>0,05)$ (Tabela 3$)$.

Tabela 3. Correlação das estações do ano em relação aos casos de doenças respiratórias.

\begin{tabular}{ccccccc}
\hline Estação & \multicolumn{2}{c}{ Belterra } & \multicolumn{2}{c}{ Itaituba } & \multicolumn{2}{c}{ Monte Alegre } \\
\cline { 2 - 7 } & $\mathrm{R}$ & $\mathrm{p}=$ valor & $\mathrm{r}$ & $\mathrm{p}=$ valor & $\mathrm{r}$ & $\mathrm{p}=$ valor \\
\hline Chuvosa & $-0,07$ & 0,2396 & 0,01 & 0,855 & 0,54 & 0,2817 \\
\hline $\begin{array}{c}\text { Menos } \\
\text { chuvosa }\end{array}$ & 0,32 & $<<0,0001$ & 0,089 & 0,1582 & 0,068 & $<<0,0001$ \\
\hline $\begin{array}{c}\text { Transição } \\
\text { C - MC }\end{array}$ & $-0,02$ & 0,6554 & $-0,115$ & 0,06716 & 0,5413 & $<<0,0001$ \\
\hline $\begin{array}{c}\text { Transição } \\
\text { MC - C }\end{array}$ & 0,05 & 0,3675 & $-0,007$ & 0,9063 & 0,068 & 0,2817 \\
\hline
\end{tabular}

Nota: $\mathrm{p}$-valor $<0,05$ significativo. $\mathrm{C}=$ Chuvoso; $\mathrm{MC}=$ Menos Chuvoso.

Santos., M., R., P., Silva., W., C., Silva., É., B., R., Gomes., A., C., S. 
$\mathrm{Na}$ análise de regressão linear, pode-se observar na Figura 6 (A e C) que os municípios de Belterra e Monte Alegre apresentaram um decréscimo considerável dos números de casos seguindo um padrão contínuo. Já a cidade de Itaituba apresentou comportamento diferente ao comparar com os demais municípios, na qual a linha de tendência sinaliza um aumento do número de casos entre 2012 e 2014, e, nos anos subsequentes decaiu consideravelmente (Figura $6 B)$.

O decréscimo observado nos municípios de Belterra e Monte Alegre podem ser explicados, possivelmente, em decorrência da adoção de medidas preventivas realizadas nos municípios em questão, por exemplo, como campanhas educativas, relacionadas a ingestão de água frequentemente ou limpeza da região nasal com solução fisiológica. Já o aumento do número de casos em Itaituba pode ter relação com a ocorrência de queimadas registradas neste município, de acordo com INPE (2021) o número dos focos de incêndio entre os anos de 2012 a 2014 alcançaram valores de $1.479 \mathrm{em}$ 2012, com decréscimo em 2013 (702 focos) e aumento considerável em 2014 (1272 focos), somando 3,453.000 neste período.
De acordo com Carmo et al. (2010) as queimadas representam cerca de 60\% do conjunto de poluentes emitidos no ar, o que provocam uma série de alterações químicas na atmosfera amazônica. Estas partículas finas produzidas pelas queimadas contribuem para $\mathrm{o}$ aumento de internações e morbidade por infecções respiratórias nos municípios localizados no arco do desmatamento da Amazônia (Mascarenhas et al. 2008).

Em um estudo realizado por Silva (2010), na cidade de Cuiabá/MT tendo como objetivo analisar os efeitos da concentração de materiais particulados, oriundos das queimadas sobre a saúde da população mais vulnerável. Observou-se que a incidência das emissões de matérias particulados estão associados às maiores ocorrências de internações por doenças respiratórias em crianças nos municípios do Estado de Mato Grosso.

Destaca-se que a diminuição do número de casos de doenças respiratórias nos municípios de Belterra e Monte Alegre, tende a ser diferente a partir de 2020, em decorrência do SARS-CoV-2, um agente viral que causa uma doença respiratória de maior gravidade, chamada COVID-19 ou coronavírus (Perlman, 2020).

A)

B)
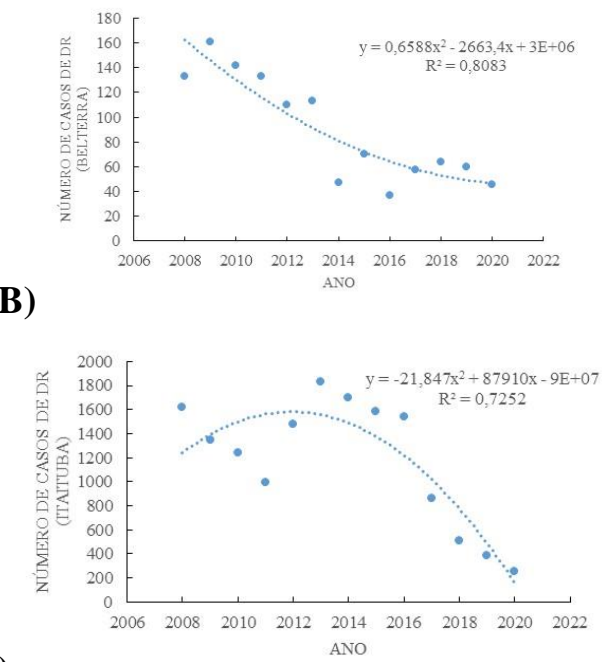

C)

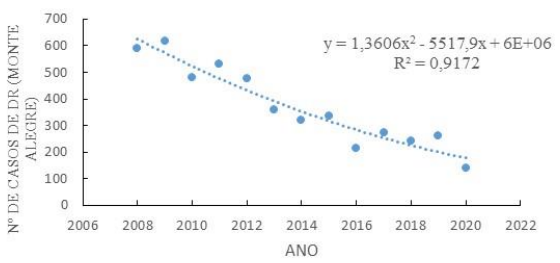

Figura 6. Dados de regressão do número de casos em função do ano em A) Belterra, B) Itaituba e C) Monte Alegre.

Santos., M., R., P., Silva., W., C., Silva., É., B., R., Gomes., A., C., S. 
O modelo de regressão múltipla via componente principal (Tabela 4) detectou que nos municípios estudados existe associação entre as condições atmosféricas e as internações por doenças respiratórias, destaca-se que o ajuste ocorreu devido a sinergia entre as variáveis meteorológicas, mas que o maior peso está relacionado a variável temperatura do ar máxima em Belterra e Itaituba e mínima em Monte Alegre (Tabela 5).

Tabela 4. Modelo de regressão múltipla via componente principal das doenças respiratórias via variáveis meteorológicas.

\begin{tabular}{lcccc}
\hline Belterra & Estimativas & Erro & Teste T & P-valor \\
\hline $\mathbf{1}^{\mathbf{a}} \mathbf{C P}$ & & & & \\
\hline Monte Alegre & 2.6982 & 0.7176 & 3.760 & $0.000212 * * *$ \\
\hline $\mathbf{2}^{\mathbf{a}} \mathbf{C P}$ & & & & \\
\hline Itaituba & 2.1935 & 0.4575 & 4.795 & $<<0,0001 * * *$ \\
\hline $\mathbf{1}^{\mathbf{a}} \mathbf{C P}$ & & & & \\
\hline
\end{tabular}

Nota: $\mathrm{p}$-valor $<0,05 \%$ possuem diferenças significativas.

O modelo de regressão utilizado via componente principal também foi o mesmo adotado nos estudos de Duarte e Mascarenhas (2007), Rosa et al., (2008) e Gomes et al. (2016) para verificar o maior peso das variáveis meteorológicas a fim identificar a variável mais influência no aumento de casos de doenças respiratórias em crianças.

Observa-se na Tabela 5 a sinergia das variáveis meteorológicas em cada componente principal e os valores respectivos da variância e de seus pesos. Destacando-se as componentes que sinalizaram associação significativa na regressão (Tabela 4). Em Belterra a $1^{\text {a }} \mathrm{CP}$ explica $\approx 85 \%$ da variabilidade do conjunto de dados, sendo o maior peso atribuído a variável temperatura máxima. Em Monte Alegre, foi a $2^{\mathrm{a}}$ $\mathrm{CP}$ que melhor se associou, apresentando $\approx 80 \%$ da variabilidade do conjunto de dados, sendo o maior peso atribuído a variável temperatura mínima e em Itaituba destacou-se a $1^{\mathrm{a}} \mathrm{CP}$ com $\approx 85 \%$ da variabilidade do conjunto de dados, sendo o maior peso atribuído a variável temperatura máxima. Com resultados diferentes ao observado neste estudo, Duarte e Mascarenhas (2007), Rosa et al., (2008) e Gomes et al. (2016) identificaram que a precipitação foi a principal análise de componente que mais contribuiu para o aumento no número de casos de internações de doenças respiratórias em diferentes municípios.

Tabela 5. Componente principal das variáveis meteorológicas.

\begin{tabular}{llllll}
\hline \multicolumn{7}{c}{ Belterra } \\
\hline Componentes: & Comp.1 & Comp.2 & Comp.3 & Comp.4 & Comp.5 \\
\hline Desvio padrão & 2.057494 & 0.66798124 & 0.43362448 & 0.33459691 & 0.143303819 \\
\hline Proporção de Variância & 0.846656 & 0.08923979 & 0.03760604 & 0.02239102 & 0.004107197 \\
\hline Proporção Cumulativa & 0.846656 & 0.93589575 & 0.97350178 & 0.99589280 & 1.000 \\
\hline Pesos & Comp.1 & Comp.2 & Comp.3 & Comp.4 & Comp.5 \\
\cline { 2 - 6 } Precipitação & 0.392 & 0.865 & 0.243 & 0.190 & \\
\cline { 2 - 5 } $\begin{array}{l}\text { Temperatura } \\
\text { MAX. }\end{array}$ & -0.468 & 0.196 & 0.735 & -0.449 & \\
$\begin{array}{l}\text { Temperatura } \\
\text { MÉDIA }\end{array}$ & -0.479 & 0.134 & & 0.213 & 0.840 \\
\hline
\end{tabular}

Santos., M., R., P., Silva., W., C., Silva., É., B., R., Gomes., A., C., S. 


\begin{tabular}{|c|c|c|c|c|c|}
\hline $\begin{array}{l}\text { Temperatura } \\
\text { MIN. }\end{array}$ & -0.438 & 0.462 & -0.660 & -0.285 & -0.278 \\
\hline $\begin{array}{l}\text { Umidade } \\
\text { Relativa }\end{array}$ & 0.453 & -0.140 & -0.682 & 0.544 & 0.115 \\
\hline \multicolumn{6}{|c|}{ Monte Alegre } \\
\hline Componentes: & Comp.1 & Comp.2 & Comp.3 & Comp.4 & Comp.5 \\
\hline Proporção Cumulativa & 0.634231 & 0.7942414 & 0.9417308 & 0.99030807 & 1.000 \\
\hline Pesos: & Comp.1 & Comp. 2 & Comp.3 & Comp.4 & Comp.5 \\
\hline Precipitação & 0.479 & 0.338 & 0.208 & 0.779 & \\
\hline $\begin{array}{l}\text { Temperatura } \\
\text { MAX. }\end{array}$ & -0.531 & -0.145 & -0.115 & 0.484 & -0.670 \\
\hline $\begin{array}{l}\text { Temperatura } \\
\text { MÉDIA }\end{array}$ & -0.331 & 0.121 & 0.931 & & \\
\hline $\begin{array}{l}\text { Temperatura } \\
\text { MIN. }\end{array}$ & -0.301 & 0.911 & -0.240 & -0.144 & \\
\hline $\begin{array}{l}\text { Umidade } \\
\text { Relativa }\end{array}$ & 0.536 & 0.140 & 0.139 & -0.359 & -0.738 \\
\hline \multicolumn{6}{|c|}{ Itaituba } \\
\hline Componentes: & Comp.1 & Comp.2 & Comp.3 & Comp.4 & Comp.5 \\
\hline Proporção Cumulativa & 0.7481578 & 0.9019306 & 0.95576822 & 0.9876681 & 1.000 \\
\hline Pesos: & Comp.1 & Comp. 2 & Comp.3 & Comp.4 & Comp.5 \\
\hline Precipitação & 0.423 & 0.473 & 0.767 & & \\
\hline $\begin{array}{l}\text { Temperatura } \\
\text { MAX. }\end{array}$ & -0.490 & -0.191 & 0.356 & -0.139 & 0.760 \\
\hline $\begin{array}{l}\text { Temperatura } \\
\text { MÉDIA }\end{array}$ & -0.487 & 0.126 & 0.289 & -0.618 & -0.531 \\
\hline $\begin{array}{l}\text { Temperatura } \\
\text { MIN. }\end{array}$ & -0.332 & 0.845 & -0.360 & 0.106 & 0.186 \\
\hline $\begin{array}{l}\text { Umidade } \\
\text { Relativa }\end{array}$ & 0.483 & 0.101 & -0.266 & -0.762 & 0.323 \\
\hline
\end{tabular}

\section{Conclusões}

Com base nas informações descritas anteriormente concluímos que nos municípios de Belterra, Itaituba e Monte alegre o número de internações por doenças respiratórias apresentou aumento no período menos chuvoso, especificamente no período de junho a agosto. Observamos que houve correlação significativa $(p<0,05)$ entre os períodos menos chuvosos com os casos de doenças respiratórias nos municípios de Belterra e Monte Alegre. Além disso, em Monte também se observou correlação no período de transição correspondente do chuvoso para menos chuvoso $(\mathrm{p}<0,05)$. Assim, por meio da PCA constatou-se que a variável temperatura máxima do ar em Belterra e Itaituba e mínima em Monte Alegre foram as que contribuíram para o aumento no número de internações por doenças respiratórias. Ademais, este estudo permitiu sinalizar que nos municípios avaliados, especialmente, nos meses de junho a agosto, apresentaram riscos do aumento de doenças respiratórias.

Diante deste cenário, devem ser adotadas medidas preventivas como a construção de políticas de saúde ambiental eficazes, a fim de valorizar a importância das pessoas no meio ambiente e a sua interação com os fatores climáticos, além dos cuidados na higiene pessoal e no ambiente doméstico evitando deixar os ambientes domiciliares fechados, uma vez que isto propicia a circulação por vírus respiratório, bem como o acesso ao conhecimento científico sobre o comportamento das variáveis meteorológicas ao longo do período mais chuvoso e menos

Santos., M., R., P., Silva., W., C., Silva., É., B., R., Gomes., A., C., S. 
chuvoso, para assim evitar a proliferação dessas enfermidades .

\section{Referências}

Alves, H.S., Freitas, A.C.V. 2021. Análise de eventos críticos de poluição atmosférica em Itabira - Minas Gerais, Brasil. Research, Society and Development, 10, 1 .

Andreão, W.L. Trindade, B.T, Nascimento, A.P, Júnior, N.C.R., Andrade, M.F., Albuquerque, T.T.A. 2019. Influence of meteorology on fine particles concentration in Vitória Metropolitan region during wintestime. Revista brasileira de meteorologia, 34(4), 459-470.

Atkinson, R.W., Strachan, D.P., Anderson, H.R., Hajat, S., Emberlin, J. 2006. Temporal associations between daily counts of fungal spores and asthma exacerbations. Occupational and Environmental Medicine, 63(9), 580-90. Disponível em: Disponível: http:// dx.doi. org/10.1136 / oem.2005.024448

Azevedo, L.H., Emberson, R.M., Esteca, F.C.N., Moraes, G.J. 2015. Macrochelid mites (Mesostigmata: Macrochelidae) as biological control agents. In: Carrillo, D., Moraes, G.J., Peña, J.E. 2015. (Eds). Prospects for biological control of plant feeding mites and other harmful organisms. Florida, Springer. 2015. pp.103-132.

Barcellos, C., Hacon, S.S. 2016. Um grau e meio. E daí?. Cadernos de Saúde Pública, 32, 1-3. Disponível em: https://doi.org/10.1590/0102311X00212315.

Briggs, D. 2003. Environmental pollution and the global burden of disease.Britishi Medical Bulletin.68, 1-24.

Carmo, C.N., Hacon, S., Longo, K.M., Freitas, S., Ignotti, E., Leon, A.P., Artaxo, P. 2010. Associação entre material particulado de queimadas e doenças respiratórias na região sul da Amazônia brasileira. Revista Panamericana de Saúde Pública, 27(1), 1016.

Carvalho, A.L.R. 2016. Manejo da via aérea para anestesia em crianças com infecção do trato respiratório superior: revisão sistemática e meta-análise para complicações perioperatórias. 2016.

César, A.C.G., Nascimento, L.F., Mantovani, K.C.C., Vieira, L.C.P. 2016. Material particulado fino estimado por modelo matemático e internações por pneumonia e asma em crianças. Revista Paulista de Pediatria, 34, 18-23. Disponível em: https://doi.org/10.1016/j.rpped.2015.06.009

Chan, P.W.K., Chew, F.T., Tan, T.N., Chua, K.B., Hooi, P.S. 2002. Seasonal variation in respiratory syncytial virus chest infection in the tropics. Pediatric Pulmonology, 34(1), 47-51. Disponível em: https://doi.org/10.1002 / ppul.10095

Conceição, R.S., Silveira, G.S.P., Veiga, A.J.P., Matta, J.M.B. 2015. A temperatura do ar e sua relação com algumas doenças respiratórias em Vitória da Conquista - BA. Revista Eletrônica Geoaraguaia, 5(2), 69 81.

Corrêa, P.B., Cândido, L.A., Souza, R.A.F., Andreoli, R.V., Kayano, M.T. 2016. Estudo do fenômeno da ilha de calor na cidade de Manaus-AM: Um estudo a partir de dados de sensoriamento remoto, modelagem e estações automáticas. Revista Brasileira de Meteorologia, 31, 167-176. Disponível em: http://dx.doi.org/10.1590/010278631220150012.

Dadbakhsh, M., Khanjani, N., Bahrampour, A., Haghighi, P.S. 2017. Death from respiratory diseases and temperature in Shiraz, Iran (2006 - 2011), International Journal of Biometeorology, 61(1), 239 246.

Dales, R.E., Cakmak, S., Judek, S., Dann, T., Coates, F., Brook, J.R., Burnett, R.T. 2003. The Role of Fungal Spores in Thunderstorm Asthma. Chest, 123(3), 745-50.

Dantas, V., Santos Silva, C., Hoelzemann, J., Campos, T., Das Chagas, G. 2016. A Influência de condições meteorológicas na concentração de gás Radônio em uma área de Mata Atlântica Dunar no Nordeste do Brasil. Revista Brasileira de Geografia Física, 9(3), 831-843. Disponível em: https://doi.org/10.26848/rbgf.v9.3.p831-843

Dapper, S.N., Spohr, C., Zanini, R.R. 2016. Poluição do ar como fator de risco para a saúde: uma revisão sistemática no estado de São Paulo. Estudos Avançados, 30, 86. DOI: https://doi.org/10.1590/S0103$\underline{40142016.00100006}$

Duarte, A.F., Mascarenhas, M.D.M. 2007. Manifestações do bioclima do Acre sobre a saúde humana no contexto socioeconômico

Santos., M., R., P., Silva., W., C., Silva., É., B., R., Gomes., A., C., S. 
da Amazônia. Amazônia: Ciência \& Desenvolvimento, 3(5), 1-14.

Fernandes, T., Hacon, S.S., Novais, J.W.Z. 2020. Variabilidade das chuvas e sua relação com os Objetivos de Desenvolvimento Sustentável em populações expostas a mudanças hídricas no sudeste da Amazônia paraense. Revista Brasileira de Geografia Física, 13(4), 1519-1536. DOI: 10.26848/rbgf.v13.4.p1519-1536

Fernandes, T., Hacon, S.S., Novais, J.W.Z., Sousa, I., Fernandes, T. 2018. Detecção e Análise de Focos de Calor no Município de Parauapebas-PA, Brasil Por Meio da Aplicação de Geotecnologia. Enciclopédia Biosfera, 15, 28. Disponível em: <http://www.conhecer.org.br/enciclop/2 018B/AGRAR/deteccao\%20e\%20analise.p df >. Acesso em: 15 mar. 2019.

Galvão, N., Leite, M.L. 2017. Relação entre variáveis climáticas e a mortalidade de idosos por doenças cardiovasculares. RBCEH, 14(3), 252-264.

Gomes, A.C.S., Costa, G.B., Vale, R.S., Santana, R.A.S., Batalha, S.S.A., Silva, J.T., Fitzjarrald, D.R. 2016. Índices micrometeorológicos e internações de crianças por doenças respiratórias em Santarém, Oeste do Pará. Ciência e Natura, 38(1), 1-6.

Gonçalves, F.L.T., Coelho, M.S.Z.S., 2010. Variação da morbidade de doenças respiratórias em função da variação da temperatura entre os meses de abril e maio em São Paulo. Ciência e Natura, 32, 103118.

Hair Junior, J.F., Anderson, R.E., Tatham, R.L. \& Black, W.C. 2005. Análise multivariada de dados. Tradução de Adonai Schlup Sant'Anna e Anselmo Chaves Neto, 5. ed. Porto Alegre: Bookman, p. 593.

Ignotti, E., Hacon, S., Junger, W.L., Mourão, D., Longo, K., Freitas, S., et al. 2010. Air pollution and hospital admissions for respiratory diseases in the subequatorial Amazon: a time series approach. Cadernos de Saude Publica, 26(4), 747-61.

INPE. 2021. Instituto Nacional de Pesquisas Espaciais. Queimadas. Disponível em: https://queimadas.dgi.inpe.br/queimadas/po rtal-static/situacao-atual/

Köppen, W., Geiger, R. 1928. Klimate der Erde. Gotha: Verlag JustusPerthes. Wall-Map $150 \mathrm{~cm} \times 200 \mathrm{~cm}$.
Lam, H.C.Y., Li, A.M., Chan, E.Y.Y., Goggins, W.B. 2016. The short-term association between asthma hospitalisations, ambient temperature, other meteorological factors and air pollutants in Hong Kong: A timeseries study. Thorax, 71(12), 1097-1109.

Liang, X., Aggarwal, R., Cherif, A., Gumel, A., Mascaro, G., Maciejewski, R. 2016. Visualizing Malaria spread under climate change. Workshop on Visualisation in Environmental Sciences, 5p.

Lima, T.F., Costa, G.F., Silva, S.M., Viana, G.S.F., Barros, B.T.D., Mendes, L.O., Castro, H.C.O., Oliveira, V.M.L.P., Marvos, W., Melo, M.K.A. 2021. Variantes climáticas e sua relação com as doenças de origem infecciosa: Uma relação integrativa. Research, Society and Development, 10(2), e7910212126. h.

Linares, D.E., Azuine, R.E., Singh, G.K. 2020. Social Determinants of Health Associated with Mental Health among U.S. Mothers with Children Aged 0-5 Years. Journal of Women's Health, 29(8), 1039-1051.

Manisalidis, I., Stavropoulou, E., Stavropoulou, A., Bezirtzoglou, E. 2020. Environmental and Health Impactos of Air Pollution: A riview. Front Public Health, 8, 14. DOI: doi: 10.3389/fpubh.2020.00014

Martins, L.O.S. et al. 2019. Gestão da Cadeia de Abastecimento de Biomassa para geração de Energia: Uma análise crítica das principais. Journal of Agricultura Science, 11(13), 253.

Mascarenhas, M.D.M., Vieira, L.C., Lanzieri, T.M., Leal, A.P.P.R., Duarte, A.F., Hatch, D.L. 2008. Poluição atmosférica devido à queima de biomassa florestal e atendimentos de emergência por doença respiratória em Rio Branco, Brasil-setembro, 2005. J Bras Pneumol, 34, 42-46.

Mold. 2008. Una breve guía para el moho la humedad y su hogar. Agency Washington, DC, United States Environmental Protection. Disponível em: http://www.epa.gov/mold/moldresources.ht ml. Acesso em:09/06/2021.

Moraes, J.F., Baniwa, A.F.M.B., Costa, F.R.C., Lima, H.P., Clement, C.R., Shepard, G.H. 2019. Historical landscape domestication in ancestral forests with nutrient-poor soils in northwestern Amazonia. Forest Ecology and Management, Amsterdam, 446, 317-330. Disponível

em: 
https://doi.org/10.1016/j.foreco.2019.04.02 $\underline{0 .}$

Moura, F.E., Nunes, I.F., Silva, G.B., Siqueira, M.M. 2006. Respiratory syncytial virus infections in northeastern Brazil: seasonal trends and general aspects. The American Journal of Tropical Medicine and Hygiene, 74(1), 165-167.

Nechet, D. 1997. Variabilidade diurna de precipitação em Belém-PA: aplicação em planejamento a médio e longo prazo. Bol Climatol, 2(3), 223-227.

Neto, E.F., Halker, E., Santos, V.J., Paiva, T.M., Neto, J.T. 2003. Influenza. Revista da Sociedade Brasileira de Medicina Tropical, 36, 267-274.

Passos, R.G., Von Sperling, M., Ribeiro, T.B. 2014. Hydrodynamic evaluation of a fullscale facultative pond by computational fluid dynamics (CFD) and field measurements. Water Science and Technology, 70(3), 569-575.

Paynter, S., Ware, R.S., Weinstein, P., Williams, G., Sly, P.D. 2010. Childhood pneumonia: a neglected, climate-sensitive disease? The Lancet, 376(9755), 1804-1805.

Perlman, S. 2020. Another Decade, Another Coronavirus. The New England Journal of Medicine, 382, 8.

Picanço, L.M.A., Gomes, A.C.S. 2019. "Variáveis Climáticas e Infecções Agudas das Vias Aéreas Superiores em Belém Do Pará"., Interfaces Científicas - Saúde e Ambiente, 7(2), 1-12. Disponível em: https://doi.org/10.17564/23163798.2019v7n2p\%25p

Porto, E.D.O., Latuf, M.O. 2017. Relações entre o comportamento climático e doenças respiratórias em Barreiras/BA. Geografia (Londrina), 26(2), 164-181.

Ribeiro, H.E., Vargas, H. 2015. Urbanização, globalização e saúde. Revista USP, 107, 13$26 . \quad$ Disponível em: https://doi.org/10.11606/issn.23169036.v0i107p13-26

Rosa, A.M., Ignotti, E., Hacon, S.S., Castro, H.A. 2008. Análise das internações por doenças respiratórias em Tangará da Serra Amazônia Brasileira. Jornal Brasileiro de Pneumologia, 34, 575-82. Disponível em: https://doi.org/10.1590/S180637132008000800006

Royé, D., Taboada, J.J., Martí, A., Lorenzo, M.N. 2015. Winter circulation weather types and hospital admissions for respiratory diseases in Galicia, Spain. International journal of biometeorology, 60(4), $507-520$.

Sales, D. M., Assis, W. L., Fonseca, B.M. 2018. Clima urbano e saúde: elementos climáticos e doenças respiratórias observadas no município de Belo Horizonte (MG) entre 2013 e 2014. Revista Brasileira de Climatologia, 14(1), 271 - 289.

Santana, E.S., Sousa, S.S., Nunes, C.S., Lima, F.L.O., Izabel, T.S.S. 2020. Principais microrganismos associados as infecções respiratórias e seus agravos a saúde: uma revisão da literatura. Research, Society and Development, 9(11), e99591110637.

Setti, L., Passarini, F., Gennaro, G., Gilio, A. Di, Palmisani, J., Buono, P., Fornari, G., Perrone, M. G., Piazzalunga, A., Barbieri, P., Rizzo, E., Miani, A. 2020. Relazione circa l'effetto dell'inquinamento da particolato atmosferico e la diffusione di virus nella popolazione. Italia: Societá Italiana di Medicina Ambientale.

Silva, A.M.C. 2010. Quantificação dos efeitos na saúde da exposição à queima de biomassa: uma contribuição ao entendimento dos efeitos da exposição ao material particulado (PM 2,5) em grupos populacionais sensíveis na Amazônia Legal. [tese] Fiocruz: Rio de Janeiro.

Silva, E.N., Ribeiro, H., Santana, P. 2014. Clima e saúde em contextos urbanos: uma revisão da literatura. Revista bibliográfica de geografía y ciencias sociales, 19, 1092.

Silva, E.N., Ribeiro, H., Santana, P. 2014. Clima e saúde em contextos urbanos: uma revisão da literatura. Revista bibliográfica de geografía y ciencias sociales. 19, 1092.

Silva, J.V.F., Silva, E.C., Silva, E.G., Ferreira, A.L., Rodrigues, A.P.R.A. 2017. Perfil da morbidade hospitalar por doenças respiratórias na infância de 0 a 9 anos na cidade de Maceió-AL no período de 2008 a 2014. Caderno de Graduação-Ciências Biológicas e da Saúde-UNIT-ALAGOAS, 3(3), 43.

Souza, A.F., Fernandes, W.A., Pavão, H.G., Albrez, G.L.E.A. 2012. Potenciais impactos da variabilidade climática sobre a morbidade respiratória em crianças, lactentes e adultos. Jornal Brasileiro de Pneumologia, 38(6), 708-715. Disponível em: 
https://doi.org/10.1590/S1806-

37132012000600005

Thomazelli, L.M. 2007. Vigilância de oito vírus respiratórios em amostras clínicas de pacientes pediátricos no sudeste do Brasil. Jornal de Pediatria, 83(5), 422-428. DOI: http://dx.doi.org/10.1590/S002175572007000600005

Valença, L.M., Restivo, P.C.N., Nunes, M.S. 2006. Variação sazonal nos atendimentos de emergência por asma em Gama, Distrito Federal. Jornal Brasileiro de Pneumologia, 32(4), 284-9. Disponível em: https://doi.org/10.1590/S1806$\underline{37132006000400005}$
Wilks, D.S. 2006. Statistical Methods in the Atmospheric Sciences. 2nd ed. 627 p. San Diego: Academic Press.

Yang, X., Lai, Y., Li, C., Yang, J., Jia, M., Sheng, J. 2020. Molecular epidemiology of Pseudomonas aeruginosa isolated from lower respiratory tract of ICU patients. 81, 2. Brazilian Journal of Biology. Doi: https://doi.org/10.1590/1519-6984.226309

Yi, L., Xu, X., Ge, W., Xue, H., Li, J., W, C., Wu, H., Liu, X., Zheng, D., Chen, Z., Liu, Q., Bi, P., Li, J. 2019. The impact of climate variability on infectious disease transmission in China: Current knowledge and further directions. Environmental Research, 173, 255-261. 